\title{
Research on the Synergy Degree Evaluation Model of Fujian Free Trade Zone and Port Logistics Based on Order Parameter
}

\author{
Shao Lijin \\ School of Economic \& Management, Fuzhou University \\ Fuzhou 350116, China \\ Minjiang University \\ Fuzhou 350108, China \\ Fuzhou Melbourne Polytechnic \\ Fuzhou 350108, China \\ E-mail: 49364408@qq.com
}

\author{
Wu Qiuming \\ School of Economic \& Management, Fuzhou University \\ Fuzhou 350116, China \\ E-mail: qiuming30@sina.com
}

\author{
Zhao Bi \\ Jiangxia University \\ Fuzhou 350002, China \\ E-mail: 357983086@qq.com
}

\begin{abstract}
The establishment of the free trade zone is a new path for China to explore the development of the economy. However, the port logistics played a very important role for free trade zone, the synergy degree between two is becoming the critical key. Based on combing and analyzing the literature review of evaluation of the synergetic development of Fujian free trade zone and port logistics, compare and analyze three kinds of synergy degree of models, and then applying the idea of order parameter of synergetics theory and the method of integration force model to build a synergy degree evaluation model of Fujian free trade zone and port logistics, put forward A new evaluation method, and analyze all the kinds of influence parameters, meanwhile the article has explained some specific problems during the process of application of the model, aiming to promote the synergetic development between the two for reference.
\end{abstract}

Keywords-Order Parameter; Fujian FTZ; Port Logistics; Synergy Degree; Integration Force

\section{INTRODUCTION}

Since Fujian was established as the core region of the Belt and Road and the second Free Trade Zones which means, the superposition of these two zones, Fujian port logistics are facing unprecedented development opportunities. Industry Development Planning of Fujian Free Trade Zone (20152019) indicated that Fujian Free Trade Zone would build a regional international shipping logistics center, with container throughputs reaching 1,700 TEU and cargo throughputs 250 billion tons. At present, Fujian Free Trade Zone includes some port areas such as Haicang Port, Dongdu Port, Jiangyin Port (No.1-9 berths), Pingtan Port, and Mawei Port with superior resources of port, shoreline and route positions. Fujian Free
Trade Zone has 67 deep water berths above 10,000 tons. Free Trade Zones benefits the attraction to inland provinces to transport the goods across the sea via Fujian by the searailway combined transportation as well as the establishment of Fujian as intermediate center of Southeast Asia, Europe and America, which indicates far-reaching and significant impact of Free Trade Zones on Fujian port logistics. Besides, the regional economic integration focusing on Free Trade Zones develops rapidely. So, "how to measure the synergy degree between them" becomes the first question demanding prompt research and solution.

\section{COMPARISON AMONG THE EXISTING THREE CATEGORIES OF SYNERGY DEGREE MODELS}

Harken, a well-known physicist, set up synergetics in 1971 and also presented the concept of synergy. In his opinion, synergy refers to cooperative behaviors that are formed by all sub-systems generating a polymerization effect stronger than single actions of each sub-system in a complex system. Degree of these cooperation behaviors is the synergy degree ${ }^{[1]}$

At present, there are many synergy degree research approaches with a broad application. According to diverse synergy (coordination) degree model measurement criteria, Dai Shuyan et al. classify them into three categories in A Study on Analytic Induction for Sustainable Development Synergy Degree Evaluation Methods, including the distance synergy degree model, the variation synergy degree model and the comprehensive synergy degree model [2]. Below, several common synergy degree models are compared according to their classification. For specifics, please see (Table 2-1).

Fund project: young and middle-aged teachers education scientific research project of Fujian province (social sciences) (JAS160412) 

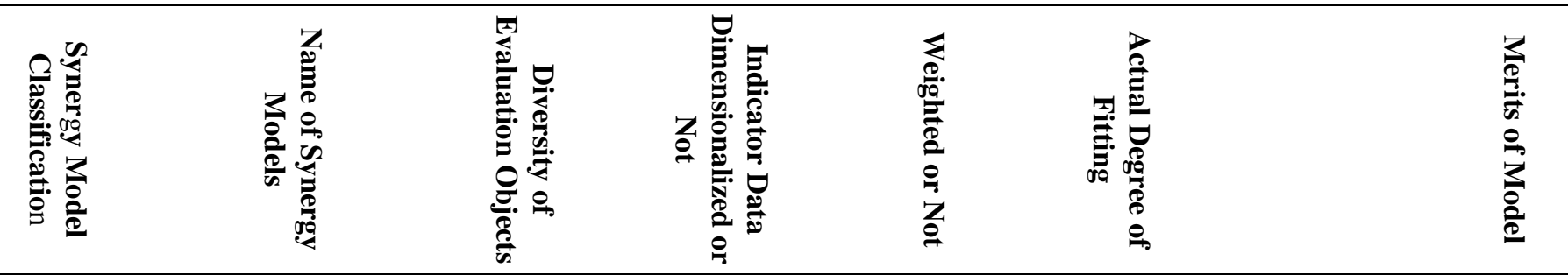

Dispersion
Coefficient
Minimization
Model

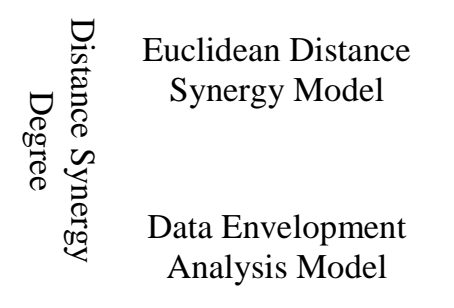

Multivariate

$\mathrm{N} \quad \mathrm{Y}$

$\mathrm{H}$ Intuitive meaning, simple
computing, free of ideal fixed evaluation variables

$\begin{array}{lll}\text { Multivariate } \quad \mathrm{N} & \mathrm{N}\end{array}$

Simple and understandable model; simple computing; and, strong applicability

Gini Coefficient
Model Binary

$\mathrm{N} \quad \mathrm{N}$

$\mathrm{L}$

Simple and understandable
model

\begin{tabular}{|c|c|c|c|c|c|c|}
\hline \multirow{2}{*}{ 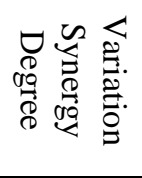 } & $\begin{array}{l}\text { Grey System } \\
\text { Theory Model }\end{array}$ & Multivariate & $\mathrm{Y}$ & $\mathrm{Y}$ & M & \multirow{2}{*}{$\begin{array}{l}\text { It studies the synergy of a } \\
\text { system dynamically and can } \\
\text { be widely applied. }\end{array}$} \\
\hline & $\begin{array}{c}\text { Order Parameter } \\
\text { Model }\end{array}$ & Multivariate & $\mathrm{Y}$ & $\mathrm{Y}$ & $\mathrm{H}$ & \\
\hline
\end{tabular}

\begin{tabular}{|c|c|c|c|c|c|}
\hline 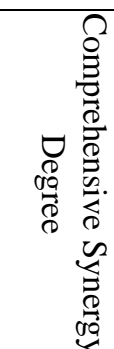 & $\begin{array}{c}\text { Energy } \\
\text { Conservation } \\
\text { Model }\end{array}$ & Multivariate & $\mathrm{Y}$ & $\mathrm{Y}$ & M \\
\hline
\end{tabular}

a. Data Source: Classified and settled by the author.

According to the above eight synergy degree evaluation models in three categories, it can be found that study objectives of most models are featured with diversity and a majority of them are adopted to evaluate their synergy degrees by describing their equilibrium or the distance from their ideal status. Additionally, evaluation approaches depend on diverse angles of evaluation to a large extent. Without any doubt, all evaluation methods have their own strengths and weaknesses, but cannot be appraised to be good or bad as they also have their own application scenarios. Therefore, it is rather difficult to find a universal application method. Based on the order parameter, the author will make use of the integrated force model proposed by Professor Wu Qiuming to construct a synergy degree evaluation model between Fujian Free Trade Zone and port logistics.

\section{FuJIAN FreE TRADE ZONE-PORT LOGISTICS SYNERGY DEGREE MODEL}

In this paper, a Fujian Free Trade Zone-port logistics synergy degree evaluation model was established based on the integrated force model and the order parameter theory of synergetics, as shown in Fig. 3-1. 


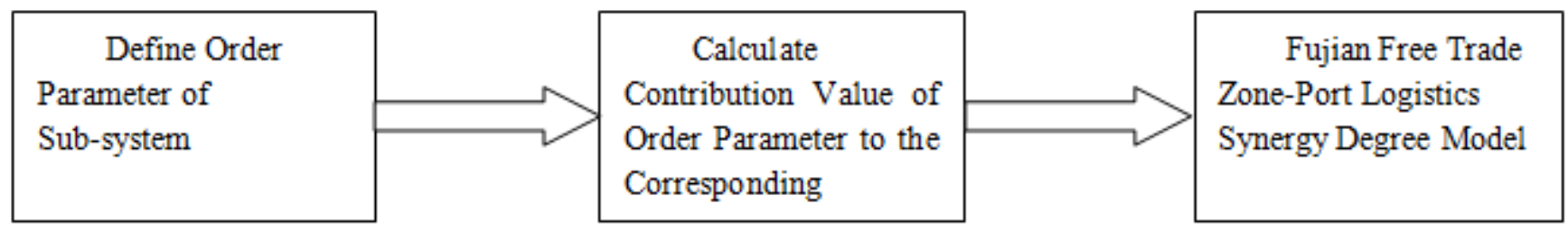

Fig. 1. Fujian Free Trade Zone-Port Logistics Synergy Degree Evaluation Model

\section{A. Establishment of an Order Parameter Indicator System}

In line with synergetics, order parameter is a parameter variable extracted from system running to reflect the overall behavior and the essential features of the system. It determines order degree and structure of the system state [1]. Therefore, selection of order parameters should observe principles of comprehensiveness, feasibility, hierarchy and dynamic and static combination. In this study, a Fujian Free Trade Zoneport logistics evaluation indicator system has been put forward based on relevant actualities and data availability of them as after integrally referring to associated research achievements and consulting scholars and experts in this field. For specifics, please see Table 3-1.

TABLE II.

An Evaluation Indicator System for Port Logistics and FuJian Free Trade Zone

\begin{tabular}{|c|c|c|c|}
\hline System & Primary Target & Order Parameter & Symbol \\
\hline \multirow{8}{*}{ Port Logistics System } & \multirow{4}{*}{ Quality Parameter (Q1) } & Cargo Throughput & $\mathrm{X} 11$ \\
\hline & & Container Throughput & $\mathrm{X} 12$ \\
\hline & & Cargo Volume of the Port & $\mathrm{X} 13$ \\
\hline & & Integrated Throughput & $\mathrm{X} 14$ \\
\hline & \multirow[b]{2}{*}{ Environment Parameter (E) } & Infrastructure Investment & Y1 \\
\hline & & Port Service Quality & $\mathrm{Y} 2$ \\
\hline & \multirow{2}{*}{$\begin{array}{l}\text { Psychological Distance } \\
\text { Parameter (d) }\end{array}$} & $\begin{array}{l}\text { Collecting and Dispatching System } \\
\text { Integrity }\end{array}$ & $\mathrm{Z} 1$ \\
\hline & & $\begin{array}{c}\text { Port Logistics Informatization } \\
\text { Degree }\end{array}$ & $\mathrm{Z} 2$ \\
\hline \multirow{7}{*}{$\begin{array}{c}\text { Economic System of } \\
\text { Fujian Free Trade } \\
\text { Zone }\end{array}$} & \multirow[t]{2}{*}{ Quality Parameter (Q2) } & Total Foreign Trade Volume & $\mathrm{X} 21$ \\
\hline & & GDP & $\mathrm{X} 22$ \\
\hline & \multirow{3}{*}{ Environment Parameter (E) } & $\begin{array}{c}\text { Ratio of Dependence on Foreign } \\
\text { Trade }\end{array}$ & Y3 \\
\hline & & The Number of Preferential Policies & Y4 \\
\hline & & Proportion of All Industries & Y5 \\
\hline & \multirow{2}{*}{$\begin{array}{l}\text { Psychological Distance } \\
\text { Parameter }(\mathrm{d})\end{array}$} & Geographic Position Superiority & $\mathrm{Z3}$ \\
\hline & & $\begin{array}{c}\text { Importance Attached to Port } \\
\text { Logistics }\end{array}$ & $\mathrm{Z4}$ \\
\hline
\end{tabular}

Data Source: Settled by the author according to related literatures.

B. Contribution Degree of Order Parameters to Corresponding Sub-systems

Dependent on the order parameter principle of synergetics, order parameter playing a leading and dominant role in the process of system evolution and development is used to represent the overall behavior of a system and a mutual competition and cooperation relation also exists among various order parameters, which further affects the system' $\mathrm{s}$ evolution course from being unordered to well-organized [8]. Regarding the contribution degree of order parameters to corresponding sub-systems, they can be figured out by an effect function and the concrete computational formula is presented as follows. 


$$
U_{i j}=\left\{\begin{array}{c}
\frac{T_{i j}-\beta_{i j}}{\alpha_{i j}-\beta_{i j}}, j \in[1, k], T_{i j} \text { With a positive effect } \\
\frac{\alpha_{i j}-T_{i j}}{\alpha_{i j}-\beta_{i j}}, j \in[k+1, \mathrm{n}], T_{i j} \text { With a negative effect }
\end{array}\right.
$$

Where, the order parameter variable is assumed as $T=\left(T_{i 1}, T_{i 2}, \ldots, T_{i n}\right)$, and $T_{i k}, T_{i 2}, \ldots, T_{i k}$ stand for positive order parameters in a direct proportion to system order degree; that is, the higher they are, the higher the degree of order will be; otherwise, the order degree of the corresponding system will be lower. $T_{i k+1}, T_{i k+2}, \ldots, T_{i n}$ refer to negative order parameters inversely proportional to the system order degree. To be specific, the higher they are, the lower the degree of order will be; otherwise, the system order degree will be higher [3]. Apparently, different order parameters have different value ranges. $\alpha_{i j}$ and $\beta_{i j}$ are maximum and minimum values of the order parameter; that is, $\alpha_{i j}=\max \left\{T_{i j}\right\}, \beta_{i j}=\min \left\{T_{i j}\right\}$ and $\beta_{i j \leq} T_{i j} \leq \alpha_{i j}$. Moreover, $j \in[1, n], i \in[1, p], n$ represents the number of order parameters and $\mathrm{p}$ is the number of sub-systems. Finally, the effect function $U_{i j} \in[0,1]$. Subsequently, the weight sum method can be employed to work out the contribution degree of each order parameter denoted as $T_{i j}$ to system orderness. The concrete computational formula is given below.

$$
U_{i=} \sum_{j=1}^{n} w_{i j} U_{i j} \sum_{j=1}^{n} w_{i j}=1
$$

Where, $U_{i}$ refers to the degree of order of each subsystem, and $w_{i j}$ to the weight of all order parameters. They can be determined by an expert scoring method and there is no need for us to go in details here.

In this paper, a synergy influence coefficient denoted by $\lambda$ was introduced to represent the magnitude of impacts of order parameters on synergy effects. It was also assumed that degrees of order were $U_{i}$ and $U_{i+1}$ respectively for two subsystems at the moment of $\mathrm{t}$; by contrast, at the moment of $\mathrm{t}=\mathrm{t} 1$, degrees of order of two sub-systems were separately $U_{i}^{1}$ and $U_{i+1}^{1}$, then,

$$
\lambda=\left\{\begin{array}{l}
1, \min \left[U_{i}^{1}-U_{i}, U_{i+1}^{1}-U_{i+1}\right] \geq 0 \\
-1, \min \left[U_{i}^{1}-U_{i}, U_{i+1}^{1}-U_{i+1}\right] \leq 0
\end{array}\right.
$$

\section{Establishment of the Synergy Degree Model}

Among diverse synergy behaviors, synergy degree can be expressed in many ways, such as dispersion, ordered variable and entropy, etc.. In this paper, the integrated force F was processed based on the integrated force model to convert it into a synergy competency index between 0 and 1 . Furthermore, it is utilized to evaluate the magnitude of synergy degree.

According to the integration theory [5], integrated force was calculated from perspectives of social system elements. The synergy of integrated force is a complicated process that can be represented by mathematical expression (3).

$$
F=E(t) \frac{Q_{1}(t) Q_{2}(t)}{d^{2}(t)}
$$

As assumed, F refers to the integrated force of synergy behaviors representing unity and integration degrees among synergy subjects. In addition, Q1 stands for the overall quality of synergy subject 1, while Q2 for that of synergy subject 2, d for psychological distance between synergy subjects and $\mathrm{E}$ for synergy environment and it also represents synergy environment coefficient $(\mathrm{E}>0)$. We also hypothesize that $\mathrm{E}=1$ for simple synergy environment, $\mathrm{E}<1$ for complex synergy environment; in other words, more complex the synergy environment is, the lower $\mathrm{E}$ will be. In addition, $\mathrm{t}$ is a time parameter. Different from the nature of integrated elements in a nature system, quality, psychological distance, environment and performance of integration elements in social system mainly composed of human beings are all functions of time, which further gives rise to time-varying internal force of integration (Chen Jiena \& Wu Qiuming, 2007) [4].

Through the above analysis, Equation (4) can be simplified correspondingly. In the case of $Q(t)=Q_{1}(t) Q_{2}(t)$ and $D(t)=\frac{1}{d^{2}(t)}$, the following synergy degree evaluation model can be obtained.

$$
C(t)=\lambda f(Q, E, D)
$$

Where, $\mathrm{C}$ is the synergy degree between two sub-systems; $\mathrm{Q}$ is the over all quality of two integrated sub-systems;

and, E stands for synergy environment coefficient, $\lambda$ for synergy influence coefficient,

D for psychological distance coefficient between two sub-systems, and t (time) for the timing of evaluation.

This function can be expressed in the following equation.

$$
C(t)=\lambda \times Q(t) \times E(t) \times D(t)
$$

By further analyzing and subdividing such three parameters that have the influence on synergy degree, following equations are gained.

$$
Q(t)=f\left(x_{1}, x_{2}, x_{3}, \cdots \cdots, x_{n}\right)
$$




$$
\begin{aligned}
& E(t)=f\left(y_{1}, y_{2}, y_{3}, \cdots \cdots, y_{m}\right) \\
& D(t)=f\left(z_{1}, z_{2}, z_{3} \cdots \cdots, z_{l}\right)
\end{aligned}
$$

In comparison with Table $4-1$, parameters $\mathrm{x} 1$ to $\mathrm{x}_{\mathrm{n}}$ refer to $\mathrm{n}$ secondary detailed indexes of the overall quality $\mathrm{Q}(\mathrm{t})$ after the integration of two sub-systems and the value of $Q(t)$ should be acquired by means of linear weighting of its secondary index scores and the corresponding index weights. Parameters from y1 to ym represent $\mathrm{m}$ secondary detailed indexes (order parameters) of the synergy environment parameter $\mathrm{E}(\mathrm{t})$; and, the value of $\mathrm{E}(\mathrm{t})$ is also obtained by linear weighting of its secondary index scores and the corresponding index weights. With regard to parameters from $\mathrm{z} 1$ to $\mathrm{zl}$, they denote $\mathrm{i}$ secondary detailed indexes (order parameters) of distance parameter $\mathrm{D}(\mathrm{t})$ between two subsystems and the value of $\mathrm{D}(\mathrm{t})$ is figured out by linear weighting of its secondary index scores and the corresponding index weights (Wu Qiuming, Zhou Bihua, Chen Jiena, 2008) [7].

It is deemed in this paper that synergy degrees and synergetic patterns of all systems at different development stages should be definitely different from each other, which has been studied by referring to national system synergism grading standards and relevant literatures. For the moment, the synergetic development degree of Fujian Free Trade Zone and port logistics is divided into five phases presented in the table below [9].

TABLE III. TABLE OF SYNERGY LEVEL AND SYNERGISTIC PATTERN CLASSIFICATION FOR FUJIAN FREE TRADE ZONE AND PORT LOGISTICS

\begin{tabular}{cccccc}
\hline $\begin{array}{c}\text { Value of } \\
\text { Synergy } \\
\text { Degree }\end{array}$ & $(-1,0]$ & $(0,0.5]$ & $(0.5,0.7)$ & $(0.7,0.9]$ & $(0.9,1)$ \\
\hline $\begin{array}{c}\text { Synergy } \\
\text { Level }\end{array}$ & Dis-synergized & Low & Medium & High & $\begin{array}{c}\text { Extremely } \\
\text { High }\end{array}$ \\
\hline $\begin{array}{c}\text { Synergetic } \\
\text { Pattern }\end{array}$ & $\begin{array}{c}\text { In Separate } \\
\text { Ways }\end{array}$ & $\begin{array}{c}\text { Traditional } \\
\text { Synergetic } \\
\text { Development }\end{array}$ & $\begin{array}{c}\text { Modern } \\
\text { Synergetic } \\
\text { Development }\end{array}$ & $\begin{array}{c}\text { Logistics- } \\
\text { oriented }\end{array}$ & Fusion \\
\hline
\end{tabular}

Data Source: Settled by the author according to national system synergism grading standards and relevant literatures.

Founded in April 2015, Fujian Free Trade Zone has a short history. Consequently, data sources of it are incomplete. Here, the author adopted a simple case to make a brief description of this model. For example, in the case that it was applied to a time synergy degree evaluation on Fujian Free Trade Zone and port logistics, we obtained an overall quality value of $6(\mathrm{Q}(\mathrm{t})$ $=6$ ), a synergy environment score of $0.6(\mathrm{E}(\mathrm{t})=0.6)$, a psychological distance value of $0.2(\mathrm{D}(\mathrm{t})=0.2)$ and a synergy influence coefficient of $0.8(\lambda=0.8)$ through relevant index system based calculations. Then, the total score of this synergy degree evaluation can be written into an equation below.

$$
C(t)=\lambda \times Q(t) \times E(t) \times D(t)=0.576
$$

By comparing with Table 3-2, the sample result indicates that a general synergy relation exists between them that fall into the category of modern synergy development pattern.

\section{Issues of Synergy Degree Model Application}

As for the above synergy degree evaluation model, it needs to be elaborated from the following aspects.

(1) Both Fujian Free Trade Zone and port logistics are social integration systems, while an integration system is an organic structure-relation combination established by various elements. This paper aims at proposing a new synergy degree evaluation approach based on order parameters and the integrated force model for social integration systems. Just as
"Entropy" is used to evaluate the synergy of social integration systems, application of "integrated force" into integration system synergy evaluation can be described as an innovative progress of the integration system oriented integration management theory. Besides, such an evaluation model serving as a general synergy degree evaluation formula is also widely applicable to social systems such as industrial clusters, virtual enterprises and virtual teams, etc...

(2) Q (t) is a comprehensive evaluation on the quality of a new system obtained by integration or combination of various sub-systems. It represents the overall quality of synergy subjects and can be defined as the summation of qualities or personality characteristics possessed by synergy subjects including individuals, groups and organizations. For such evaluations, it is an outcome of overall quality product of all sub-systems. At the time of selecting particular indexes, special attention should be paid to how to assign them in line with proper reasons.

(3) $\mathrm{E}$ (t) ranging from 0 to 1 is a parameter used to evaluate the synergy environment of a system. Acting as a medium parameter for the space between sub-systems, it covers a large area including hard and soft environment. In short, they are physical and mental environment. Regarding an equation of synergetic integrated force, $\mathrm{E}$ is the synthesis of all objective factors and conditions that affect the existence of organizations, involving leadership environment, institutional environment, policy environment, investment environment, living environment, sociocultural environment, infrastructure environment, market supply-demand environment, traffic network environment and information network environment, etc.. 
(4) d (t) is an evaluation on the psychological distance between sub-systems. In the model, $\mathrm{D}(\mathrm{t})$ refers to reciprocal of $d(t)$ square, which makes the equation much simpler. However, value of it has a great influence on the final result of this equation. For this reason, its value should be assigned properly and range between 0 and 1 . Such an evaluation is primarily carried out by qualitative indexes and largely depends on subjective assessments. In the domain of psychology, psychological distance is defined as the "emotional bonding degree between human beings in the process of interpersonal communications" (Wu Qiuming, Bai Liying, 2015) [6]. However, when psychological distance is brought into a synergetic integration environment, it refers to "emotional tacit understanding degree among different synergy subjects" and it is a synthetic distance perception generated by joint actions of multiple factors. Furthermore, such a distance perception makes it more difficult for both parties of synergy to closely cooperate with each other. Principally, it consist of geographical (spatial) distance, communication smoothness, consistency or similarity among value orientation, strategy, objective, vision and culture, etc., information symmetry degree and trust, etc..

(5) Synergy influence factor $\lambda$ represents the influence degree of order parameters on synergy effects. As it is a value figured out based on the contribution of order parameters to the system order degree, $\lambda$ can be positive or negative with an absolute value less than 1 accordingly.

(6) What needs to be particularly illustrated is that such an evaluation model mainly relies on statistical indexes to perform analysis and calculations and it thus should be analyzed and demonstrated qualitatively and quantitatively. Basic thoughts are as follows. First, characteristic variables of integration system synergy should be found and then used to represent the consistency of internal psychological identification and order of extrinsic behaviors of an integration system. Second, after indexes affecting the synergy degree of an integration system have been found and clustering analysis performed, corresponding results should be aggregated by virtue of $\mathrm{E}, \mathrm{Q}$ and $\mathrm{d}$; in other words, these results serve as secondary or three-level indexes of E, Q and d, so that the model can be applied for data operation. Moreover, it still needs to be emphasized that indexes selected are representative and data are available and comparable.
Undoubtedly, raw data should be also non-dimensionalized in this process as units of different indexes are different.

\section{CONCLUSION}

This article constructed the synergy evaluation model of Fujian Free Trade Zone and port logistics based on the order parameter, proposing a new evaluation method. This model can objectively and systematically evaluate the development level so it is the basis to realize the sustainable development, with a theoretical and practical significance to adjust and upgrade the industrial structure within Fujian Free Trade Zone, promote the service quality and level and finally realize an integrative development. Due to the length of this article and the short establishment time of the Fujian Free Trade Zone, the available data is limited. So the specific application of this model including the collection of the indicators and data as well as the analysis and computation process will be given in the subsequent papers.

\section{REFERENCES}

[1] H.Haken.Advanced Synergetics[M].Translated by Guo Zhian.Beijing : Science Press, 1989 : 210-238.

[2] Dai Shuyan,Huangxinjian.Analysis of Evaluation Methodology of Sustainable Development ' $s$ Synergy Degree[J].Science and Management,2004(6) : 22-24.

[3] He Yude,Ma Zujun.Analysis of Synergy Degree of Regional Logistics and Economy in the Industrial Transfer-Based on Empirical Research in Sichuan[J].Modern Management,2014(1) : 99-101.

[4] Chen Jiena, $\mathrm{Wu}$ Qiuming.Interpretation of Integration Theory of Industrial Cluster[J].Scientific Progress and Strategies,2007,24(3) : 5861.

[5] Wu Qiuming.Theory of Integrated Management[M]. Beijing: Economic Science Press, 2004.

[6] Wu Qiuming,Bai Liying.Integration of Human and Organizations : From P-O Bilateral Matching to Influence of Psychological Distance[J].Journal of Beijing Institute of Technology(Social Science Edition),2015,17(4) : 105-110.

[7] Wu Qiuming,Zhou Bihua,Chen Jiena.Evaluation Model of "Field Force" of Industrial Cluster and Parameters Analysis[J].Studies in Science of Science, 2008,26(s1) : 65-69.

[8] Sun jianjing. Science and technology, economic and social coordinated development model research [J]. China Journal of management science, 1996, (2) : 13-18.

[9] li zhaoguo, wang ting. Research on the development of port logistics in fujian province and fujian - asean trade cooperation [J]. Logistics engineering and management, 2015(11):60-61. 